\title{
MULTI LAYERS SUPPLY CHAIN MODELING BASED ON MULTIAGENTS APPROACH
}

\author{
Samia Chehbi, Yacine Ouzrout, Aziz Bouras \\ \{samia.chehbi, youzrout, abouras\}@univ-lyon2.fr
}

FRANCE

\begin{abstract}
This paper proposes a strategic multi layers model based on multi agents approach for supply chain system. It introduces a formulation and a solution methodology for the problem of supply chain design and modeling. In this paper we describe and analyze the relationships among main entities of a supply chain, such as suppliers, producers, and distribution centers, in the aim to design the agents and define their behavior. We also study, how these relationships can be formulated in a multi layer model. Finally, a generic multi agent model is illustrated.
\end{abstract}

\section{INTRODUCTION}

The most popular research topic in the field of supply chain (SC) management is the formulation of strategic and efficient model. This can be opted by different manners, by using artificial intelligence tools or integer programming methods ( $\mathrm{Wu}, 2001)$. The problem is commonly arises in the evaluation of some parameters characterizing SC state. A number of production producers supply a collection of distribution centers with multiple products, which, in turn, supply customers with specified demand quantities of different products. The challenge is to determine the number, location, capacity, and type of convenient actors to minimize the total cost of the SC. The mathematical problem of formulation in production context exists since a long time where some works, like the one of Goeffrion and Graves (Goeffron, 1972) described a multi-commodity single-period production-distribution problem and solved it by Benders Decomposition. Recently, Hong Y. et al. (Hong, 2003) has developed a proved method based on constraints to design a strategic production-distribution model. Other works have been published recently under this theme, like (Dong, 2003), where Dong J. et al. analyze the formulation and design a demonstrated mathematical model based on lemmas and theorems. Most efforts in these works consider SC activities separately and proceed by studying SC as a linear model and try to represent it globally. We provide another view to model SC, considering it as a non linear system with a high level of complexity, and we try to apply technical tools, classically used to resolve complex systems design and 
modeling. In the next sections, we describe briefly some SC features, then, we detail our formulation steps and describe the parameters, variables and constraints used to design the multi layers system. After, we proceed by giving some key issues in SC design and modeling using multi agent systems and finally, we give an example of dimensioning supply chain problem.

\section{FORMULATION OF SUPPLY CHAIN ORGANISATION}

Today, competition in global markets with heightened expectations of customers has pushed the enterprises to invest and make more importance of their SCs. Consequently, to reduce cost and improve service levels, effective SC strategies must take into account interactions at the various levels in the SC. To improve their SC performance, firms must focus on understanding most information and relationships nature of all the partners and SC actors. Having an idea of a model projecting their $\mathrm{SC}$, these firms can improve many strategic decisions like forecasting operations, predicting customers' demands and decreasing warehouse stocks costs.

This paper shows how theoretical programming formulations can be applied to SC design problem, and focuses on the fact of dividing SC into upstream and downstream parts by considering the manufacturer as the reference mark. The main objective in the proposed approach is to evaluate an objective function (to maximize) and a cost function (to minimize). We assume that the SC's flow concerns a single family of product.

Actors in classical SC models (Simchi-Levi, 2000) are not organized, and interactions are ignored. This leaded us to propose in a precedent work (Chehbi, 2003) a multi-layers reorganization in order to facilitate the SC evaluation's phase. Hence, any SC can be transformed into a multi-layers architecture (figure 1).

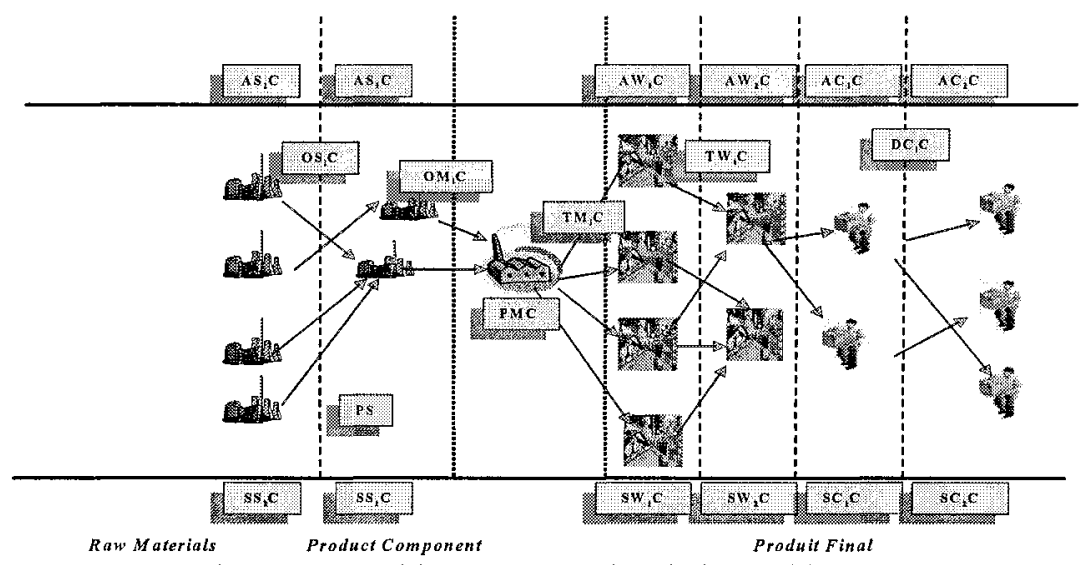

Figure 1 - Multi Layers Supply Chain Architecture

\subsection{Supply Chain Parameters}

Before formulating the model, we introduce the basic parameters notations and definitions. In this study, we use the following indices: $s \in S$, a set of candidate 
suppliers; $M$ the single manufacturer; $w \in W$, a set of $\mathrm{w}$ arehouses and distribution centers; $c \in C$, a set of customers; $p c \in P C$, a set of product components needed for production; $p \in P$, the single type of product characterizing the SC. Using these notations, we describe some considered costs as follows:

Added Cost: It is the cost obtained when introducing an actor in the chain. Hong et al. (Hong, 2003) used the same principle in their formulation of a logical constraints model for SC. They call it the fixed cost to open and operate an actor. Each actor has its proper added cost in the SC. Relating to each actor type, we distinguish: $A S i C$ (Added Supplier ' $i$ ' Cost), AWiC (Added Warehouse ' $i$ ' Cost) and ACiC (Added Customer ' $i$ ' Cost).

Action Costs: Signifies the internal cost evaluated for each actor. We distinguish two types of costs; the first is the Production Cost of one unit of the final product in the case of the main manufacturer $(P M C)$, or a unit of a product component in the case of a supplier $P S_{i}$. The second type concerns the Storage Cost of a unit of the final product or its components $\left(\mathrm{SS}_{\mathrm{i}} \mathrm{S}\right.$ for suppliers, $\mathrm{SW}_{\mathrm{i}} \mathrm{C}$ for warehouses and $S S_{i} C$ for customers).

Interaction Costs: Interactions between supply chain actors play an important role in the total cost. Along the upstream supply chain, we define TMiC (Transportation Cost Between the manufacturer and its ${ }^{\prime \text { th }}$ customer), TWiC (Transportation Cost between warehouses) and DCiC (Distribution Cost between customers). In the downstream chain, we define $O M i C$ as the cost of materials ordered by the main manufacturer to its $i^{\text {th }}$ supplier and OSiC between manufacturers to deliver the product.

\subsection{Evaluation of objective function}

Before providing the total cost function, we define other notations related to each actor location in the multi layer architecture. A supplier $i$ located in a level $n$ is indicated by $S_{L n}^{n}$ with $L_{n}$ the number of suppliers in the layer $n$. The same rule is applied to the other actors, so we have as a result the indices matrix (Supply Chain Matrix) described below.

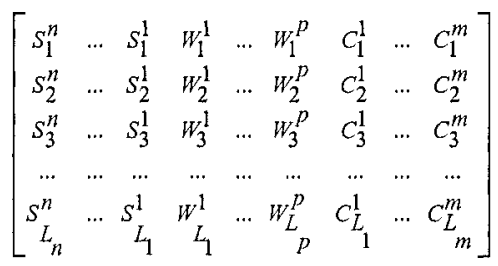

Using these notations and all parameters evolved before, we give the total cost of supply $\mathrm{c}$ hain as the sum of $a d d e d$, action and interaction costs. B y e vidence, the total added cost is the sum of added supplier costs, added warehouses costs and added supplier costs.

Total Added Supplier Cost $=\sum_{i=1}^{L 1} A S i C+\sum_{i=1}^{L 2} A S i C+\ldots+\sum_{i=1}^{L n} A S i C$, Total Added Warehouse Cost $=\sum_{i=1}^{L 1} A$ AWC + $\sum_{i=1}^{L 2} A$ AWiC $+\ldots+\sum_{i=1}^{L p} A$ ATiC, and Total Added Customer Cost $=\sum_{i=1}^{L 1} A C i C+\sum_{i=1}^{L 2} A C i C+\ldots+\sum_{i=1}^{L m} A C i C$ 
After evaluating added costs of each type of supply chain actors, we sum them to have the total added cost in the chain. To evaluate total action cost, it is important to notice that there are probably actors which are not producers or storage centers; we interpret this by introducing a binary coefficient $q_{L j}^{i}$ to the supplier $i$ belonging to the $j^{\text {th }}$ layer, whether it is a producer $\left\{q_{L j}^{i}=1\right\}$ or not $\left\{q_{L j}^{i}=0\right\}$.

Total Production Cost $=$ Production Costs of suppliers + Production Cost in the main manufacturer and Total Storage Cost $=$ Storage Cost of suppliers + Storage Cost of warehouses + Storage Cost of customers.

Total Action Cost $=\sum_{i=1}^{L 1} P S_{i} \cdot q_{L 1}^{i}+\sum_{i=1}^{L 2} P S_{i} q_{L 2}^{i}+\ldots+\sum_{i=1}^{L n} P S_{i} q_{L n}^{i}++P M C+\sum_{i=1}^{L 1} S S_{i} C \cdot q_{L 1}^{i}+\sum_{i=1}^{L 2} S S_{i} C q_{L 2}^{i}+\ldots$
$+\sum_{i=1}^{L n} S S_{i} C q_{L n}^{i}+\sum_{i=1}^{L 1} S W_{i} C \cdot q_{L 1}^{i}+\sum_{i=1}^{L 2} S W_{i} C q_{L 2}^{i}+\ldots+\sum_{i=1}^{L p} S W_{i} C q_{L p}^{i}+\sum_{i=1}^{L 1} S C_{i} C \cdot q_{L 1}^{i}+\sum_{i=1}^{L 2} S C_{i} C q_{L 2}^{i}+\ldots+\sum_{i=1}^{L m} S C_{i} C q_{L m}^{i}$

Total Action Cost $=$ Sum of ordered costs between suppliers + sum of ordered costs between the main manufacturer and its s uppliers + sum of transportation costs between the main manufacturer and its customers + sum of transportation costs between warehouses + sum of distribution costs between customers.

$$
\begin{aligned}
& \text { Total Interactin Cost }=\sum_{i=1}^{L 2} O S{ }_{i} C+\ldots+\sum_{i=1}^{L n} O S{ }_{i} C+\sum_{i=1}^{L 1} O M_{i} C+\sum_{i=1}^{L 1} T M_{i} C+\sum_{i=1}^{L 2} T W_{i} C+\ldots \\
& +\sum_{i=1}^{L p} T W W_{i} C+\sum_{i=1}^{L 1} D C{ }_{i} C+\sum_{i=1}^{L 2} D C{ }_{i} C+\ldots+\sum_{i=1}^{L(m-1)} D C{ }_{i} C
\end{aligned}
$$

By consequence, the objective function is given by: Min $F=$ Total Added Supplier Cost + Total A dded Warehouse Cost + Total Added Customer Cost + Total Action Cost + Total Interaction Cost.

\section{MULTI AGENT SYSTEMS MODELING}

This section presents an issue for modeling the dynamic behavior of the proposed $\mathrm{SC}$ multi layers model. Our aim is that to obtain an efficient tool of simulation which can be applied to quantify the flow of SC information. With this described model, we think be capable to determine strategic policies are effective in smoothing and reducing variations in the SC. In most recent works in this topic, SC and enterprises networks have been a fertile area of multi agent simulations. That's because there is a growing need to developing decentralized efficient tools aiding to more performed management tools.

Referring to (Ferber, 1995), a multi agent system is a collection of, possibly heterogeneous, computational entities, having their own goals and problem-solving capabilities. Won et al. (Dong, 2002) suggest a set of interactive agents for Harbor SC network. Lin et al. (Lin, 1998) present multi agents architecture to model and simulate SC information system, they propose a shared environment based on agents simulating orders processes. Researches on agents-based SC management can be divided into three types: (1) A gent-based a rchitecture for coordination, (2) a gentbased simulation of SCs and (3) dynamic formation of SCs by agents. Our current work is a combination between the two first types of researches. It proposes an agent-based architecture doted of decision making agents to insure collaboration between SC parts. Based on various designs for multi agent systems in the literature (Dong, 2002) and many previous r esearches, we try to design a $\mathrm{n}$ a gent-based S C 
model, described statically and dynamically via three types of agents (figure 2). An agent type called 'controller' to model the SC dimensioning, a set of agents to model SC dynamic, divided into physical agents representing tangible existing objects (such as wholesalers, customers, etc) and logical agents defining a virtual agent for each layer. They are doted of information functions used to control the information flows and manage the interactions as described bellow:

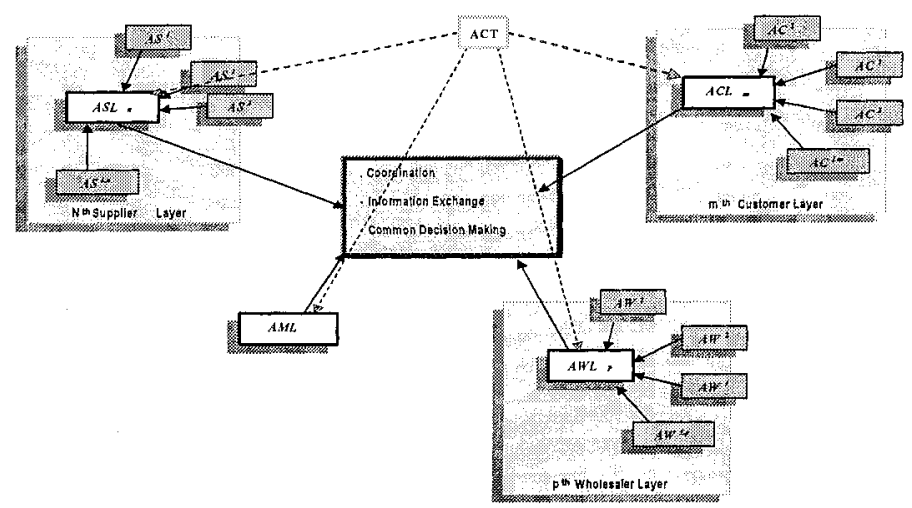

Figure 2 - Multi-Layers Agent SC Model

\subsection{Agent-Actors}

For each SC entity, we define a specified agent. So, $A C_{j}^{i}$ means the $i^{\text {th }}$ customer agent in the $j^{\text {th }}$ customer layer. Applying the same signification, we have $A S_{j}^{i}$ for suppliers and $A W_{j}^{i}$ for wholesalers. They are designed with classical standardized functionalities to communicate, negotiate and send or receive requests from other agent-actors of the same layer and the agent-layer (see figure 3, (a)). Three principle modules are implemented in the agent-actor; each one insures a specific task: The communication module serves as a reception-sending filter with the other agents of the same layer. There may be several types of information exchanged, such as products demand, negotiation messages, asking for a shared information, etc. The knowledge management module contains all parameters perceived by the agent and a part of the database. For example, action costs are the most important variables existing in the internal database. Moreover, this agent has the possibility of asking for external data from other agents. To coordinate between previous modules, a coordination module is added.

\subsection{Agent-Layer}

Each layer is managed by a logical cognitive agent responsible for reactive agents' management in its same layers. We define one agent manager for the manufacturer called $A M L$ (Agent Manufacturer), one another for each customer layer $\left(A C L_{i}\right)$, one agent for each layer $\left(A S L_{i}\right)$ and also for each wholesaler layer $\left(A W L_{i}\right)$. It has to insure and maintain the minimization cost of its layer when it receives the order from the controller agent (Figure 3, (b)). Interactions between these agents enable the flows of products and information within a layer and to other layers that are 
immediately adjacent to it in the SC. In addition to contain the same modules implemented in the Agent-Actor, we define the decision making module designed to propose negotiated decisions to Agents-Actors. Its internal data base comprises interaction and added costs related to its layer.

\subsection{Agent-Controller}

We define one controller-agent $(A C T)$ in the system, designed to evaluate strategic decisions for SC dimensioning. All formulation part is implemented in the decision making module of this agent. It must communicate continuously with all agentslayers of all the chain in order to update its information (figure 3, (c)).
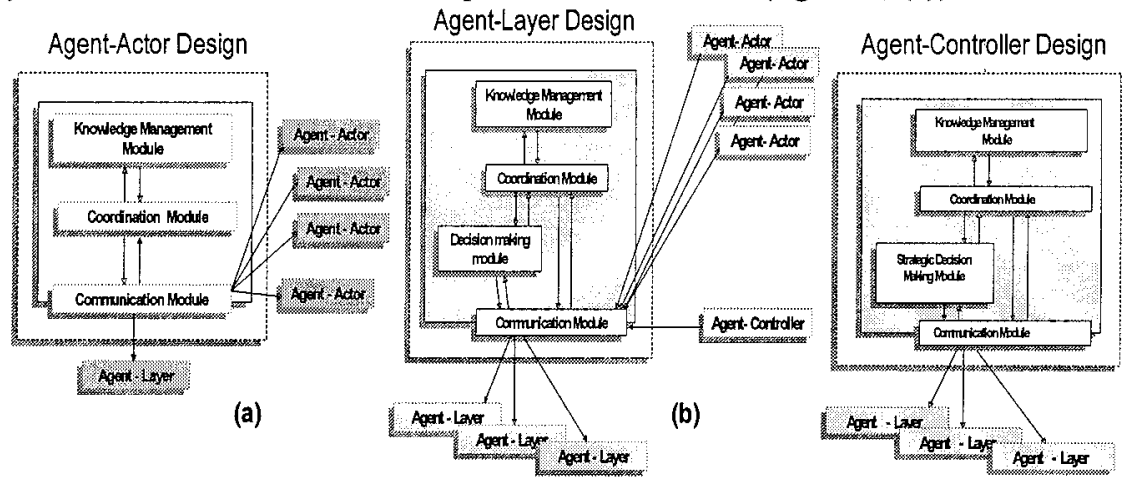

Figure 3 - Agents design

\section{ILLUSTRATIVE EXAMPLE}

In this section, we present an example of a simplified supply chain illustrated in (figure 4). The chain is related to the product $P$, assembled and delivered by the main manufacturer Man. In the manufacturing process, we distinguish an intermediate product component $I P$ assembled and delivered by a secondary center of assembly $D$. There are three first suppliers $\{A, B, C\}$ for three raw materials $\{M l$, $M 2, M 3$ \}, one storage center $E$ for the product component and the raw material $M 3$ in addition to three storage centers $\{G, L, M\}$ for the final product $P$. We also have six final customers $\{N, O, P, Q, R, S\}$.

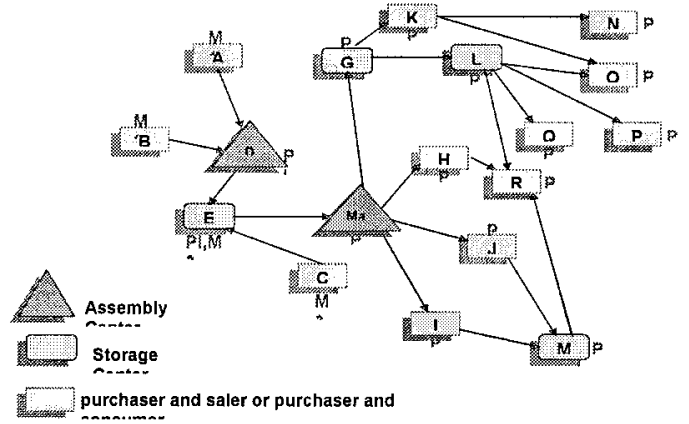

Figure 4-SC description - Illustrative Example 


\subsection{Multi Agent Model}

In order to decompose the chain into layers, it is important to extract a hierarchic tree to distinguish and define the various levels. We take as reference mark the manufacturer of the final product and then we advance in the hierarchy in the two directions (customers and suppliers) (figure 5). In this example we can divide the chain into six layers $\{S 1, S 2, S 3, C 1, C 2, C 3\}$.

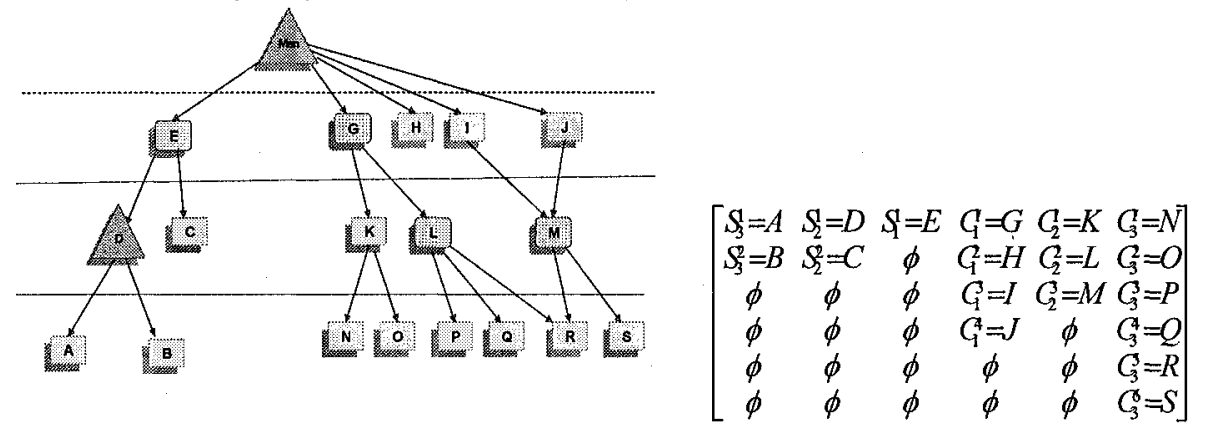

Figure 5-Hierarchical tree

Each element in the matrix is represented by one reactive agent containing its actor's information, and each column in the matrix is represented by one cognitive agent manager to coordinate between layers and make partial common decisions, in addition to another cognitive agent defined to take strategic decisions for supply chain dimensioning. By consequence, our multi agent organization contains seven reactive agents related to six cognitive agent-managers in each layer and one cognitive agent-controller. In order to clarify the use of each agent, we describe a simple example of a scenario showing the problem of supply chain dimensioning.

\subsection{Dimensioning problem description}

We assume that the main manufacturer Man is located in France; its storage center $G$ is located in USA and it has to know if it is profitable to open a secondary center of assembly in USA instead of delivering products to USA with a high cost of transportation. We suppose that in the state $S t_{l}$, the agent controller $A C$ has estimated the total cost of the chain at a value $C S t_{l}$. We suppose also that the state of the chain in the case of adding the secondary center of assembly in USA is called $S t_{2}$, were the manufacturer buys product components $\{P I, M 3\}$ in USA and assembles them in the added center (figure 6).

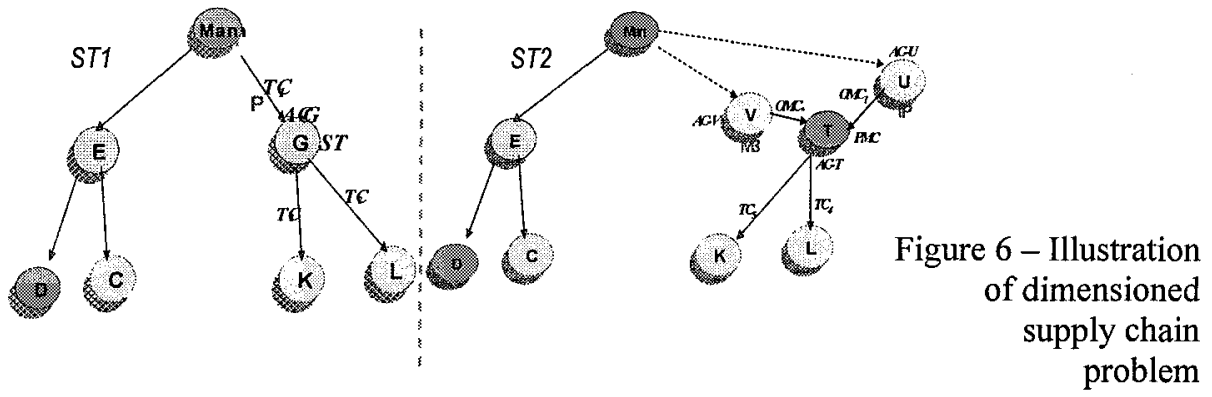


Each agent-actor sends to the agent-manager of the specific layer the values of estimated added costs $A C$ of the actors $\{U, V, T\}$ respectively, in addition to the production cost of the secondary manufacturer $T\{P M C\}$. The agents-managers, in turn, send these information in addition to the interaction costs $\left\{O M C_{1}, O M C_{2}, T C_{4}\right.$, $\left.T C_{5}\right\}$ to the agent controller. This one collects all cost values, calculates the total cost $C S t_{2}$, compares with the previous cost $C S t_{1}$ and finally decides which state is profitable for the manufacturer; the state $S t_{1}(C S t 1<C S t 2)$ or $S t_{2}\left(C S t_{1}>C S t_{2}\right)$.

\section{CONCLUSION}

Although there is a wealth of literature and research on modeling of strategic SC design, there is an apparent lack of theoretical consideration of SC constraints. In this present paper, we formulate a strategic SC model based on ordered layers and including pertinent constraints. We have considered the SC with a high complexity, in order to justify our choice of strategy in designing it as complex system. We have expressed relationships among actors via inter-relations between layers. We have extensively discussed our parameters and variables representation. We presented our multi layers model as a parallel organization of non linear sub systems of layers where interactions. In addition, we propose a multi agent issue for SC modeling. This paper aims at introducing a representation for building a mathematical model based on the constructive idea in constructing a multi layers model. The principle in designing this model is that to minimize global system cost while satisfying all customers' demands and to design learned agents to simulate SC environment and their actors' behavior. We think our work can open a novel way in proceeding of SC problem resolve by using decentralized tools.

\section{REFERENCES}

1. Chehbi S, Derrouiche R, Ouzrout Y, Bouras A. Multi Agent Supply Chain Architecture to Optimize Distributed Decision Making. The 7th World Multiconference on Systemic, Cybernetics and Informatics (SCI), Floride-USA. 2003.

2. Dong J, Zhang D. Multitiered Supply Chain Networks: MultiCriteria Decision-Making under Uncertainty. New-York-USA. 2003.

3. Dong W, Hie S.K, Nak H. K. Combined Modeling with Multi Agent System and Simulation: It's Application to Harbor Supply chain Management.The 35th Annual Hawaii International Conference on System Sciences (IEEE). USA, 2002.

4. Ferber J. 'Les Systèmes Multi Agent: Vers une Intelligence Collective'. Inter Edition, Paris, 1995.

5. Geoffrion A.M, Graves G.W. An Interactive Approach for Multi-Criterion Optimization, with an Application to the Operation of an Academic Department. Management Science, 19(4) :357-368, 1972.

6. Hong Y, Zhenxin Y, T.C. Edwin C. A strategic Model for Supply Chain Design with Logical Constraints : Formulation and Solution'. Computer \& Operations Research, 30(14) :2135-2155, 2003.

7. Lin F, Shaw M. J. Reengineering the Order Fulfilment Process in Supply Chain Networks. International Journal of Flexible Manufacturing Systems, (10:3), pp.197-229, 1998.

8. Simchi-Levi D, Kaminiski P, Simchi-Levi E. Designing and Managing the Supply Chain: Concepts, Strategies, and Case Studies. International Editions, 2000.

9. Wu T.T, O' Grady P. A Network Based Approach to the Design of Supply Chains. Arizona-USA, 2001. 